\title{
Second primary malignancy risk among patients with gastric cancer: a nationwide population-based study in Taiwan
}

\author{
San-Chi Chen ${ }^{1}$ - Chia-Jen Liu ${ }^{1,2,3} \cdot$ Yu-Wen $\mathrm{Hu}^{2,3,4}$ - Chiu-Mei Yeh ${ }^{5}$. \\ Li-Yu Hu ${ }^{6}$ - Yen-Po Wang ${ }^{3,7}$ • Yi-Ping Hung ${ }^{1}$ C Cheng-Hwai Tzeng ${ }^{1,3}$. \\ Tzeon-Jye Chiou ${ }^{1,3,8}$ • Tzeng-Ji Chen ${ }^{3,5}$ • Chung-Jen Teng ${ }^{2,3,9}$
}

Received: 21 October 2014/Accepted: 24 February 2015/Published online: 13 March 2015

(c) The International Gastric Cancer Association and The Japanese Gastric Cancer Association 2015

\begin{abstract}
Background Several studies have reported an increase in second primary malignancies (SPMs) among gastric cancer patients.

Methods Patients who were newly diagnosed with gastric cancer between 1997 and 2011 were recruited from the Taiwan National Health Insurance database. Those who had antecedent malignancies or gastrointestinal stromal tumor were excluded. Standardized incidence ratios (SIRs) of SPMs were calculated. Risk factors for cancer development were analyzed by Cox proportional hazards
\end{abstract}

S.-C. Chen and C.-J. Liu contributed equally to this manuscript.

Chung-Jen Teng

cjteng.tw@gmail.com

1 Division of Hematology and Oncology, Department of Medicine, Taipei Veterans General Hospital, Taipei, Taiwan

2 Institute of Public Health, National Yang-Ming University, Taipei, Taiwan

3 School of Medicine, National Yang-Ming University, Taipei, Taiwan

4 Cancer Center, Taipei Veterans General Hospital, Taipei, Taiwan

5 Department of Family Medicine, Taipei Veterans General Hospital, Taipei, Taiwan

6 Department of Psychiatry, Kaohsiung Veterans General Veterans Hospital, Kaohsiung, Taiwan

7 Endoscopy Center of Diagnosis and Treatment, Taipei Veterans General Hospital, Taipei, Taiwan

8 Division of Transfusion Medicine, Department of Medicine, Taipei Veterans General Hospital, Taipei, Taiwan

9 Division of Hematology and Oncology, Department of Medicine, Far Eastern Memorial Hospital, No. 21, Sec. 2, Nanya S. Rd., Banciao Dist, 22060 New Taipei City, Taiwan models. Effects of treatments for gastric cancer were treated as time-dependent variables.

Results During the 15-year study period, 47,729 gastric cancer patients were recruited. Overall, 2,110 SPMs developed during a total follow-up of 137,798 person-years. The SIR for all cancers was 1.46. The SIRs for specific follow-up periods were $1.43,1.41$, and 1.21 at $>10$ years, 5-10 years, and 1-5 years, respectively. After excluding SPMs that developed within 1 year, significantly higher SIRs were seen for cancers of the head and neck (1.34), esophagus (2.16), colon and rectum (1.37), bones and soft tissues (1.95), ovaries (2.89), bladder (1.47), or kidneys (1.44), as well as non-Hodgkin's lymphoma (5.56). Multivariate analysis showed that age $\geq 70$ years [hazard ratio (HR) 1.19], being male (HR 1.37), diabetes mellitus (HR 1.30), chronic obstructive pulmonary disease (HR 1.17), and liver cirrhosis (HR 1.94) were independent risk factors. Radiotherapy (HR 1.24) and chemotherapy (HR 1.87) were independent risk factors, but surgery (HR 0.67) was not.

Conclusions Patients with gastric cancer are at increased risk of developing SPM. Close surveillance of patients with risk factors over a longer period should be considered.

Keywords Gastric cancer - Second primary malignancy Population-based study

\section{Introduction}

Gastric cancer is the fourth most common cancer in the population worldwide. Its incidence is decreasing in Western countries; over $70 \%$ of new cases are occurring in developing countries [1]. In Taiwan, gastric cancer mortality has shown a decreasing trend in the past few decades [2], a trend similar to those seen in Japan [3] and Korea [4], 
the two nations with the highest gastric cancer incidences in Asia [5]. There are a number of reasons for the increased survival of gastric cancer patients, for instance more hygienic strategies for food conservation, increased screening using upper gastrointestinal panendoscopy, better surgical techniques, improved lymph node dissection, and advances in adjuvant chemotherapy and radiotherapy [6]. However, the risk of second primary malignancy (SPM) is of concern because patients with SPMs present lower survival rates than those without [7-9].

Several single institutes in Asia have reported that SPMs occur in gastric cancer patients at incidences of 0.9-3.4\% [9-11]. However, when group comparisons are performed, the relative risk or incidence ratios observed in large-scale ( $>5,000$ patients) studies are inconsistent. The incidence of SPM at specific sites was found to be relatively high in a Japanese cancer registry study [12] and a Korean singlehospital case series [7], but not in another multicenter case series [13]. A population-based study in Sweden showed a high standardized incidence ratio (SIR) of SPM after gastric cancer, but this increase may have been caused by closer surveillance (i.e., surveillance bias) [14]. So far, no nationwide population-based studies on this topic in Asia have been reported. Thus, given the improvement in gastric cancer survival, a nationwide population-based study of SPM in gastric cancer patients in Taiwan was felt to be a worthwhile pursuit.

The National Health Insurance Research Dataset (NHIRD) in Taiwan possesses a nationwide population dataset for health research. All malignancies are registered precisely, meaning that the NHIRD is suitable for analyzing SPM. Besides age and sex, the NHIRD also includes complete information on major surgeries, chemotherapy, radiotherapy, and comorbidity-information that was not simultaneously integrated into most previous studies on gastric cancer. The aim of the study described in the present paper was to use the NHIRD to compare the overall incidence of SPM in gastric cancer patients with the expected incidence in an age-, sex-, and calendar-year-matched population. In addition, we investigated the potential predisposition of gastric cancer patients for chemotherapy, radiotherapy, and comorbidity.

\section{Materials and methods}

\section{Data sources}

Taiwan's National Health Insurance (NHI) program is a mandatory universal health insurance program that began in 1995. It supplies comprehensive medical care to all Taiwan's residents, with a coverage rate of more than $99 \%$ [15]. This program provides coverage for outpatient, emergency, inpatient, dental, and traditional Chinese medicine services, as well as prescription drugs. Our NHI program also allows all cancer patients to easily acquire treatment and surveillance after being diagnosed with cancer at a reasonably low cost, no matter what their socioeconomic background. The so-called "catastrophic illness" system exempts patients from copayment under the NHI program. Any patient certified for catastrophic illness can get appropriate outpatient follow-up, including all the necessary clinical tools, such as physical examinations, laboratory tests, endoscopies, and imaging studies such as a computed tomography (CT) scan and/or a magnetic resonance imaging (MRI) scan, for \$NT 100 dollars ( US\$ 3.2 or $¥ 370$ ).

Our study used the NHIRD database, which is managed by the National Health Research Institute of Taiwan and available publicly. The NHI Database of Catastrophic Illness provides comprehensive information on the NHI enrollment of and health care resources for all patients with severe diseases. It integrates several NHI databases, including claims data, NHI enrollment files, and the registry for drug prescriptions. Gastric cancer and all other types of malignancies are categorized as catastrophic illnesses. Because of the possibility of exempting patients from most copayments, the insurance office strictly reviews the diagnosis. Registration as a cancer patient often requires pathological proof of the diagnosis. When there is no pathological confirmation, the clinical diagnosis must be supplemented by persuasive results from laboratory tests and imaging studies. In addition, since the referral system in Taiwan has not been fully enforced, patients with cancers of any stage or type can easily obtain access to all levels of hospitals, including medical centers for diagnosis and management-implying that they must be registered. Accordingly, the accuracy of cancer diagnoses from the catastrophic illness data is reliable, and the vast majority of all cancer patients in Taiwan are included in the data.

All information that could potentially identify an individual patient is encrypted. These data are confidential, as mandated by the Bureau of National Health Insurance and the National Health Research Institute. Because the NHI dataset contains unidentifiable secondary data for research purposes, our study is exempt from full review by the institutional review board of Taipei Veterans General Hospital (2013-10-002CE).

\section{Study population}

We conducted a nationwide population-based cohort study spanning January 1, 1997 to December 31, 2011. Newly diagnosed gastric cancer cases were retrieved from the Registry of Catastrophic Illness and diagnosed based on the International Classification of Diseases, Ninth Revision, 
Clinical Modification (ICD-9-CM) code 151. Patients diagnosed with gastric cancer before January 1, 1997 were not enrolled. Patients aged $<20$ years at the time of diagnosis and those who had antecedent malignancies or gastrointestinal stromal tumor were excluded. Data on comorbidity and gastric cancer treatments such as surgery, radiation, and chemotherapy were collected for further analysis. The surgery was defined as curative gastrectomy.

\section{Statistical analyses}

The main dependent variable was development of SPM. Subjects identified from the Registry for Catastrophic Illness required histological evidence of malignant diagnosis. These patients were followed until the occurrence of SPM, death, or dropout from the NHI program before the end of the year 2011.

This study used the SIR, which is defined as the observed number of cancer occurrences divided by the expected number, to determine the risk of SPM among patients in this study cohort. The expected number of cancers was calculated by simply multiplying the cancer incidence in the general population by the number of patients in this study cohort who were in the corresponding age group. Each group was stratified according to sex, calendar year, and age in 5-year intervals by the corresponding stratum-specific person-time accrued in the cohort. The incidence of cancer among the general population was acquired from the Taiwan National Cancer Registry. The $95 \%$ confidence intervals (CIs) of the SIRs were estimated according to the assumption that the observed number of cancers followed a Poisson probability distribution. We defined the SIRs for subgroups based on sex and age group. A subgroup analysis stratified by the time taken to develop the SPM was carried out to avoid surveillance bias. For the same reason, SIRs for different types of cancers were estimated by excluding SPMs that occurred within 1 year after the diagnosis of gastric cancer. Risk factors for the development of SPM in gastric cancer patients were analyzed using univariate and multivariate Cox proportional hazards models. We analyzed factors including not only age, sex, and comorbidities but also surgery, chemotherapy, and radiotherapy. Treatments after gastric cancer were treated as time-dependent variables to prevent immortal time bias. Factors with $p<0.1$ in univariate analyses were included in the Cox multivariate analysis.

Data were extracted and computed using the Perl programming language (version 5.12.2; Perl Foundation, Walnut, CA, USA). Data linkage, processing, and sampling were conducted using Microsoft SQL Server 2008 (Microsoft Corp., Redmond, WA, USA). SAS 9.2 software (SAS Institute Inc., Cary, NC, USA) was used for all statistical analysis. Statistical significance was defined as $p<0.05$.

\section{Results}

\section{Characteristics of the study population}

We identified 50,598 patients with gastric cancer in the NHIRD's Catastrophic Illness Registry from 1997 to 2011. Among these, 25 patients were under the age of 20;2,158 patients had antecedent malignancies before gastric cancer, 23 patients were lost to follow-up, and 663 patients were misclassified (see the flow chart in Fig. 1). Therefore, the final sample consisted of 47,729 patients, including 30,415 $(63.7 \%)$ males and 17,314 (36.3\%) females, with a median age of 69 years at diagnosis (interquartile range, 57-77 years). Overall, this cohort was observed for 137,798 person-years from 1997 to 2011. The characteristics of this cohort are shown in Table 1.

\section{All cancers}

During the observation period, 2,045 cancers developed. In comparison with the general population, patients with gastric cancer had a significantly increased risk of any type of cancer (SIR 1.46; $95 \%$ CI 1.40-1.52, $p<0.001$ ). The SIR was $1.38(95 \%$ CI 1.31-1.46, $p<0.001)$ for men and 1.67 (95\% CI 1.54-1.80, $p<0.001$ ) for women. A subanalysis showed that patients who were relatively young at the time of diagnosis had the highest SIR for any cancer. Patients aged 20-39 had an SIR of 7.59 (95\% CI 5.29-10.56). Subgroup analysis based on the time taken to develop SPM after the diagnosis of gastric cancer showed that the SIRs for $0-1,1-5,5-10$, and $>10$ years after the diagnosis of gastric cancer were 1.98 (95\% CI 1.83-2.13, $p<0.001), 1.21$ (95\% CI 1.12-1.30, $p<0.001), 1.41$ (95\% CI 1.29-1.54, $p<0.001)$, and 1.43 (95\% CI $1.21-1.69, p<0.001)$, respectively. The results of this subanalysis are summarized in Table 2.

\section{Specific cancer types}

After excluding SPMs that developed within 1 year after the diagnosis of gastric cancer, significantly higher SIRs were observed for cancers of the head and neck (1.34, $95 \%$ CI 1.09-1.63), esophagus (2.16, $95 \%$ CI 1.61-2.84), colon and rectum $(1.37,95 \%$ CI 1.21-1.54), bones and soft tissue $(1.95,95 \%$ CI 1.11-3.16), ovaries $(2.89,95 \%$ CI 1.71-4.57), bladder (1.47, $95 \%$ CI 1.16-1.84), kidneys (1.44, $95 \%$ CI 1.06-1.93), as well as non-Hodgkin's lymphoma $(5.56,95 \%$ CI 4.68-6.55). SIRs for specific cancer types are shown in detail in Table 3. 
Fig. 1 Flow chart for patient inclusion in the study
From January 1, 1997 to December 31, 2011

50,598 patients with newly diagnosed gastric cancer

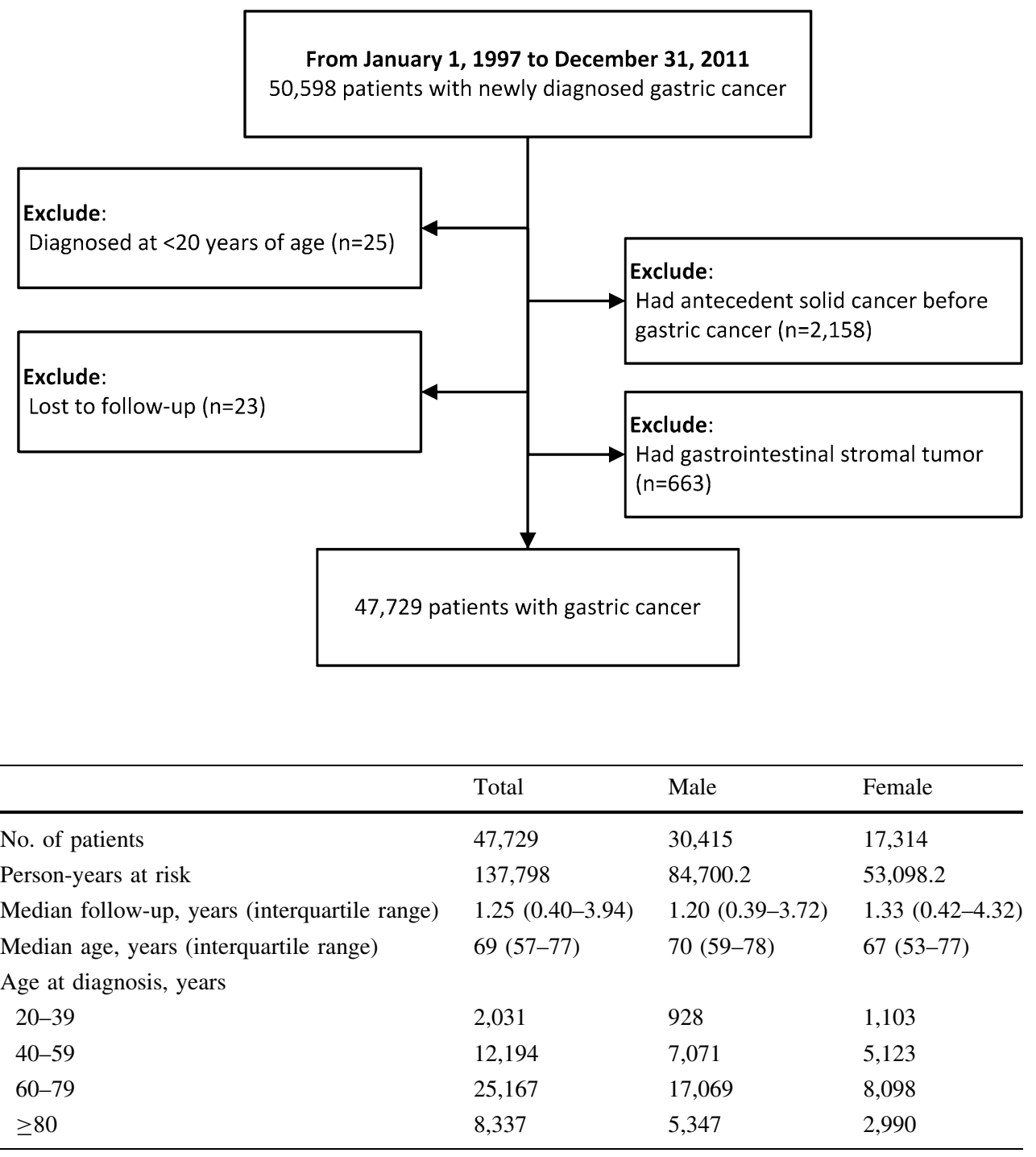

\begin{tabular}{llll}
\hline & Total & Male & Female \\
\hline No. of patients & 47,729 & 30,415 & 17,314 \\
Person-years at risk & 137,798 & $84,700.2$ & $53,098.2$ \\
Median follow-up, years (interquartile range) & $1.25(0.40-3.94)$ & $1.20(0.39-3.72)$ & $1.33(0.42-4.32)$ \\
Median age, years (interquartile range) & $69(57-77)$ & $70(59-78)$ & $67(53-77)$ \\
Age at diagnosis, years & & & \\
20-39 & 2,031 & 928 & 1,103 \\
$40-59$ & 12,194 & 7,071 & 5,123 \\
$60-79$ & 25,167 & 17,069 & 8,098 \\
$\geq 80$ & 8,337 & 5,347 & 2,990 \\
\hline
\end{tabular}

Table 1 Characteristics of patients with gastric cancer

Table 2 Standardized incidence ratios according to sex, age at diagnosis, and follow-up time after gastric cancer diagnosis

\begin{tabular}{|c|c|c|c|c|c|c|c|c|c|}
\hline \multirow[t]{2}{*}{ Characteristics } & \multicolumn{3}{|l|}{ Total } & \multicolumn{3}{|l|}{ Male } & \multicolumn{3}{|l|}{ Female } \\
\hline & Observed & Expected & $\operatorname{SIR}(95 \% \mathrm{CI})$ & Observed & Expected & $\operatorname{SIR}(95 \% \mathrm{CI})$ & Observed & Expected & $\operatorname{SIR}(95 \% \mathrm{CI})$ \\
\hline All cancers & 2,045 & $1,401.42$ & $1.46(1.40-1.52)$ & 1,405 & $1,017.10$ & $1.38(1.31-1.46)$ & 640 & 384.31 & $1.67(1.54-1.80)$ \\
\hline \multicolumn{10}{|c|}{ Age at diagnosis, years } \\
\hline 20-39 & 35 & 4.61 & $7.59(5.29-10.56)$ & 15 & 1.68 & $8.93(5.00-14.73)$ & 20 & 2.93 & $6.82(4.17-10.54)$ \\
\hline $40-59$ & 456 & 155.13 & $2.94(2.68-3.22)$ & 268 & 88.58 & $3.03(2.67-3.41)$ & 188 & 66.56 & $2.82(2.44-3.26)$ \\
\hline $60-79$ & 1178 & 879.38 & $1.34(1.26-1.42)$ & 850 & 657.68 & $1.29(1.21-1.38)$ & 328 & 221.70 & $1.48(1.32-1.65)$ \\
\hline$\geq 80$ & 376 & 362.29 & $1.04(0.94-1.15)$ & 272 & 269.17 & $1.01(0.89-1.14)$ & 104 & 93.13 & $1.12(0.91-1.35)$ \\
\hline \multicolumn{10}{|c|}{ Follow-up period after gastric cancer diagnosis, years } \\
\hline $0-1$ & 662 & 334.90 & $1.98(1.83-2.13)$ & 457 & 246.35 & $1.86(1.69-2.03)$ & 205 & 88.55 & $2.32(2.01-2.65)$ \\
\hline $1-5$ & 746 & 616.81 & $1.21(1.12-1.30)$ & 512 & 449.96 & $1.14(1.04-1.24)$ & 234 & 166.85 & $1.40(1.23-1.59)$ \\
\hline $5-10$ & 495 & 350.69 & $1.41(1.29-1.54)$ & 338 & 251.89 & $1.34(1.20-1.49)$ & 157 & 98.80 & $1.59(1.35-1.86)$ \\
\hline$\geq 10$ & 142 & 99.01 & $1.43(1.21-1.69)$ & 98 & 68.91 & $1.42(1.15-1.73)$ & 44 & 30.11 & $1.46(1.06-1.96)$ \\
\hline
\end{tabular}

SIR standardized incidence ratio, $C I$ confidence interval 


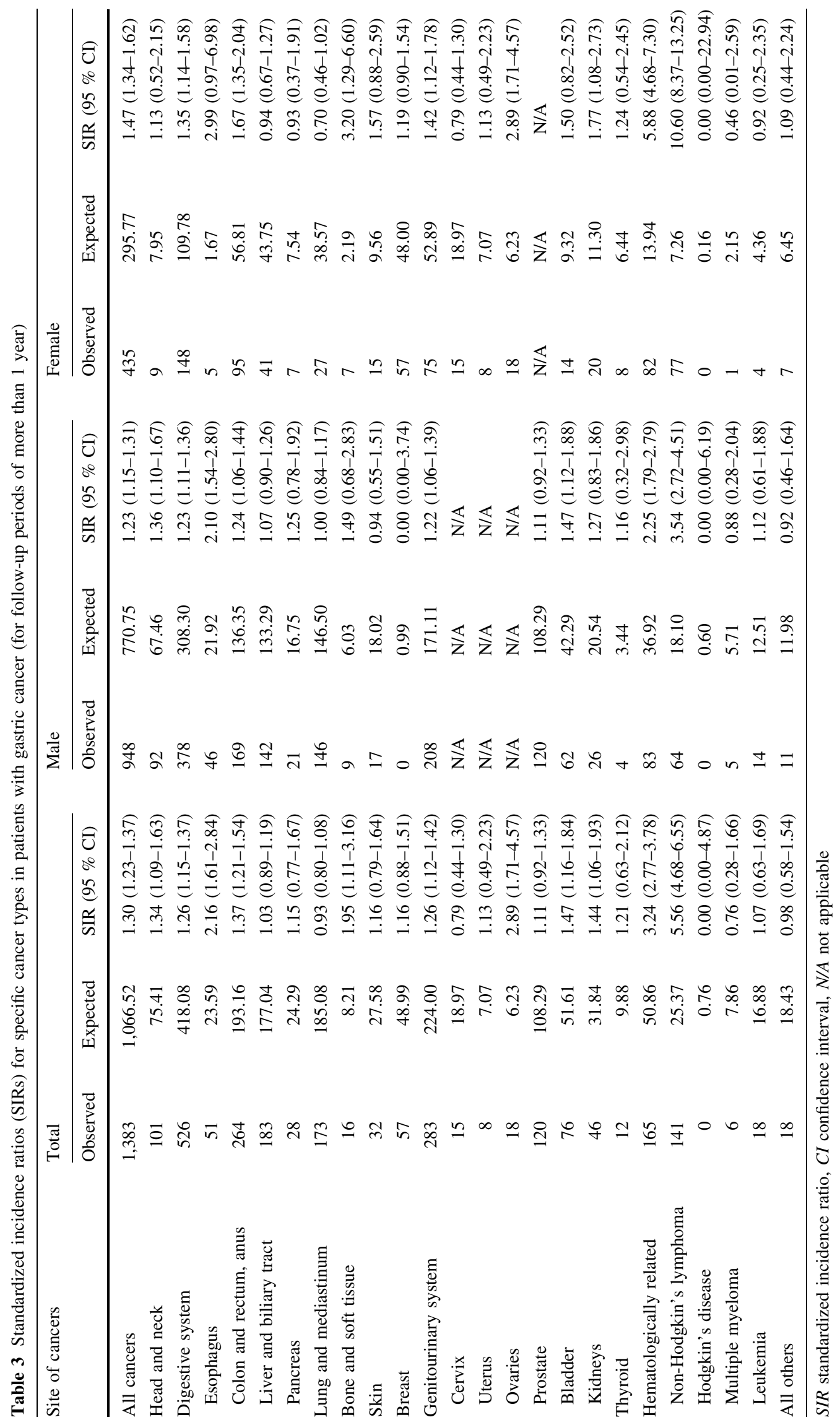


Table 4 Risk factors for SPM development in patients diagnosed with gastric cancer (for follow-up periods of more than 1 year $)(n=26,420)$

\begin{tabular}{|c|c|c|c|c|}
\hline \multirow[t]{2}{*}{ Variables } & \multicolumn{2}{|l|}{ Univariate analysis } & \multicolumn{2}{|c|}{ Multivariate analysis $^{\mathrm{a}}$} \\
\hline & $\operatorname{HR}(95 \% \mathrm{CI})$ & $p$ value & $\mathrm{HR}(95 \% \mathrm{CI})$ & $p$ value \\
\hline Age $\geq 70$ & $1.26(1.13-1.40)$ & $<0.001$ & $1.19(1.06-1.34)$ & 0.002 \\
\hline Sex (male) & $1.42(1.26-1.59)$ & $<0.001$ & $1.37(1.22-1.54)$ & $<0.001$ \\
\hline \multicolumn{5}{|l|}{ Comorbidities } \\
\hline Diabetes mellitus & $1.34(1.18-1.52)$ & $<0.001$ & $1.30(1.15-1.48)$ & $<0.001$ \\
\hline COPD & $1.32(1.16-1.49)$ & $<0.001$ & $1.17(1.02-1.33)$ & 0.020 \\
\hline Liver cirrhosis & $2.15(1.71-2.71)$ & $<0.001$ & $1.94(1.54-2.45)$ & $<0.001$ \\
\hline Autoimmune diseases & $1.22(0.97-1.53)$ & 0.093 & $1.14(0.90-1.44)$ & 0.284 \\
\hline Dyslipidemia & $1.09(0.94-1.26)$ & 0.246 & & \\
\hline ESRD & $1.18(0.87-1.59)$ & 0.283 & & \\
\hline \multicolumn{5}{|l|}{ Treatment $^{\mathrm{b}}$} \\
\hline Surgery & $0.73(0.63-0.84)$ & $<0.001$ & $0.67(0.58-0.78)$ & $<0.001$ \\
\hline Chemotherapy & $1.25(1.13-1.39)$ & $<0.001$ & $1.24(1.10-1.39)$ & $<0.001$ \\
\hline Radiotherapy & 2.01 (1.69-2.39) & $<0.001$ & $1.87(1.56-2.24)$ & $<0.001$ \\
\hline
\end{tabular}

\section{Predictors of SPM risk}

Univariate Cox proportional hazards analysis (see Table 4) showed that age $\geq 70$ years, being male, diabetes mellitus, COPD, liver cirrhosis, and autoimmune disease were significantly associated with a higher risk of SPM development. Multivariate analysis showed that age $\geq 70$ years [hazard ratio (HR) 1.19, $95 \%$ CI 1.06-1.34, $p=0.002$ ], being male (HR 1.37, $95 \%$ CI 1.22-1.54, $p<0.001$ ), diabetes mellitus (HR 1.30, $95 \%$ CI 1.15-1.48, $p<0.001$ ), COPD (HR 1.17, $95 \%$ CI 1.02-1.33, $p=0.020$ ), and liver cirrhosis (HR 1.94, $95 \%$ CI $1.54-2.45, p<0.001)$ remained independent predictors of SPM development. Risk of second primary cancer varied depending on the treatment instigated for gastric cancer, i.e., surgery (HR $0.67,95 \%$ CI $0.58-0.78, p<0.001$ ), radiotherapy (HR 1.24, $95 \%$ CI $1.10-1.39, p<0.001$ ), or chemotherapy (HR 1.87, $95 \%$ CI 1.56-2.24, $p<0.001$ ).

\section{Discussion}

This is the first nationwide population-based study in Asia to clearly demonstrate a significantly increased SIR of metachronous SPM in patients with gastric cancer. Our main findings are that (1) gastric cancer patients have a significantly increased risk of SPM in cancer of the head and neck, esophagus, colon and rectum, bones and soft tissues, ovaries, bladder, or kidneys, as well as non-Hodgkin's lymphoma, and (2) the independent risk factors for SPM include age $\geq 70$ years, being male, diabetes mellitus, COPD, liver cirrhosis, chemotherapy, and radiotherapy.
Previous studies have reported incidences of SPM following gastric cancer ranging from 0.9 to $9.5 \%[7-14,16-$ 22]: $0.6-6.7 \%$ for metachronous [7-10, 16-21] and $0.6-5.4 \%$ for synchronous [8-11] SPM. Most of these studies were single-institute case series; only a few were nationwide or multiple-institute cohort studies that included a reference group to test the SIR or relative risk of SPM [7, 12-14]. Those cohort studies reported that cooccurrence of second primary cancer (i.e., within 6 months of the initial cancer) occurred in $0.9-1.0 \%$ of the gastric cancer patients who underwent gastrectomy [9-11], and two other population-based studies also reported similar findings: in those studies, the incidence of another cancer in the first year after the diagnosis of gastric cancer was 1.1 [14] and $0.15 \%$ [12]. For this reason, we excluded the SPMs found during the first year of follow-up after diagnosis of gastric cancer $(n=662$ accounting for $1.39 \%$ of all the gastric cancer patients in our cohort), but the results of the analysis remained unchanged (SIR 1.30, $95 \% \mathrm{CI}$ 1.23-1.37). Our results are highly credible due to our unbiased nationwide selection of patients, who were diagnosed based on reliable diagnostic criteria with histological evidence.

Because the NHI's database is so comprehensive, the follow-up period was long as 15 years. Our study was the first to show that the SIR of SPM was highest at $>10$ years follow-up (SIR 1.43), followed by 5-10 years (SIR 1.41), and then 1-5 years (SIR 1.21). In the subgroup analysis, we confirmed that those aged 20-39 years at diagnosis had the highest risk of SPM (SIR 7.59). Both Hiyama et al. [12] and the SEER database [23] obtained similar findings for younger patients. This implies that patients with gastric 
cancer, especially those aged 20-39 years and those who have survived for more than 10 years (accounting for $7.6 \%$ in our study cohort), are still at risk for SPM, and a circumspect follow-up and screening strategy is necessary for these patients.

Similar to the Japan Cancer Registry study, our findings showed an increased SIR of SPM, including head and neck and gastrointestinal malignancies [12]. Our data also showed significantly higher SIRs for non-Hodgkin's lymphoma (5.56) and cancers of the bone and soft tissues (1.95), ovaries (2.89), kidneys (1.44), and the bladder (1.47). It is not uncommon for gastric MALT lymphoma to occur after gastric cancer, since these two malignancies share a common etiology: H. pylori infection [24, 25]. To avoid clerical error, we excluded all cases with gastric lymphoma and gastrointestinal stromal tumors (GISTs), but the correlation between SPM of non-Hodgkin's lymphoma and gastric cancer remained. A reasonable possibility is a late effect of systemic chemotherapy [26, 27] or regional radiotherapy, or both [27]. Mauch reported that treatment with radiotherapy may pose an excessive risk of secondary non-Hodgkin's lymphoma (as high as 10.6 per 10,000 person-years), and the risk may be even higher (up to 16.1) with radiotherapy plus chemotherapy [27]. Secondary bone and soft tissue sarcomas have been widely reported after solid tumor therapies during childhood [2831 ], and they have been attributed to multiple treatment modalities and a long latency period. However, since cancers of the bone and soft tissue are relatively rare events (estimated at $0.07 \%$ in our cohort), the exact relationship and underlying mechanism merit further exploration and study.

Regarding multivariate analysis, this is the first study to demonstrate that comorbidities such as diabetes mellitus, COPD, and liver cirrhosis are independent risk factors for SPM after diagnosis of gastric cancer. In the general population, there is a higher overall risk of cancer in patients with diabetes [32], of non-liver malignancies in patients with cirrhosis [33], and of nonpulmonary malignancies in patients with COPD [34]. Furthermore, old age and being male are also risk factors, which is consistent with previous findings $[9,10,18]$. Follow-up and clinical decision-making for patients with these risk factors should be more aggressive.

Our study discovered that surgery (HR 0.67) lowers the risk of SPM while radiotherapy (HR 1.87) and chemotherapy (HR 1.24) increase it; all of these factors were regarded as time-dependent variables. Patients who received surgery for gastric cancer might have had earlier stages of the disease, which implies that the more advanced the gastric cancer, the more frequently SPM occurs. In addition, fewer patients who received surgery for gastric cancer might have undergone cytotoxic therapy. Some studies have also shown that adjuvant chemotherapy may not increase the incidence of SPM [35-37]. Those studies were all from single institutes with relatively few SPM events $(<200)$, so they may not have had the power to detect the impact of chemotherapy. Since they are derived from a nationwide gastric cancer cohort with long-term follow-up periods of up to 15 years, our results may serve to inform clinicians that these patients should be given optimal adjuvant therapy but should not be overtreated [35].

This retrospective study has several limitations. First, family history and several lifestyle factors such as obesity, tobacco use, and alcohol use may serve as potential confounders, and these factors were not included in the NHI database. However, we found that some comorbidities which are independent risk factors for SPM may be associated with such lifestyle factors. Second, the disease extent (i.e., stage) and microscopic features of the gastric cancer were not recorded in the NHI database. Nevertheless, about $80 \%$ of the patients underwent surgery in our study, which means that most of the gastric cancers were operable. The relation between gastric cancer stage and SPM is unknown, although Kim [9] proposed that earlystage gastric cancer is a predictive factor for SPM. Finally, clinical symptoms and laboratory data were not available, so we were not able to analyze the predictive value of specific clinical situations.

In conclusion, we have demonstrated that the risk of SPM is significantly higher in gastric cancer patients: as survival and follow-up increases, SIR increases. Age, being male, comorbidity, chemotherapy, and radiotherapy are independent risk factors. Hence, after the first 5 years of surveillance for gastric cancer, longer, closer surveillance might be necessary for early detection of SPM.

Acknowledgments This study was supported by a grant from Taipei Veterans General Hospital (V103B-022 and V103E10-001), and is based in part on data from the National Health Insurance Research Database, provided by the Bureau of National Health Insurance, Department of Health and managed by National Health Research Institutes. The interpretation and conclusions contained herein do not represent those of the Bureau of National Health Insurance, Department of Health or National Health Research Institutes.

Conflict of interest The authors declare no conflict of interest.

\section{References}

1. Jemal A, Bray F, Center MM, Ferlay J, Ward E, Forman D. Global cancer statistics. CA Cancer J Clin. 2011;61:69-90.

2. Chen CJ, You SL, Lin LH, Hsu WL, Yang YW. Cancer epidemiology and control in Taiwan: a brief review. Jpn J Clin Oncol. 2002;32(Suppl):S66-81.

3. Tanaka K, Kiyohara Y, Kubo M, et al. Secular trends in the incidence, mortality, and survival rate of gastric cancer in a 
general Japanese population: the Hisayama study. Cancer Causes Control. 2005;16:573-8.

4. Choi Y, Gwack J, Kim Y, et al. Long term trends and the future gastric cancer mortality in Korea: 1983-2013. Cancer Res Treat. 2006;38:7-12.

5. Wu CY, Wu MS, Kuo KN, Wang CB, Chen YJ, Lin JT. Effective reduction of gastric cancer risk with regular use of nonsteroidal anti-inflammatory drugs in Helicobacter pylori-infected patients. J Clin Oncol. 2010;28:2952-7.

6. Otsuji E, Yamaguchi T, Sawai K, Hagiwara A, Taniguchi H, Takahashi T. Recent advances in surgical treatment have improved the survival of patients with gastric carcinoma. Cancer. 1998;82:1233-7.

7. Kim JY, Jang WY, Heo MH, et al. Metachronous double primary cancer after diagnosis of gastric cancer. Cancer Res Treat. 2012;44:173-8.

8. Yoshino K, Asanuma F, Hanatani Y, Otani Y, Kumai K, Ishibiki $\mathrm{K}$. Multiple primary cancers in the stomach and another organ: frequency and the effects on prognosis. Jpn J Clin Oncol. 1985;15(Suppl 1):183-90.

9. Kim C, Chon HJ, Kang B, et al. Prediction of metachronous multiple primary cancers following the curative resection of gastric cancer. BMC Cancer. 2013;13:394.

10. Eom BW, Lee HJ, Yoo MW, et al. Synchronous and metachronous cancers in patients with gastric cancer. J Surg Oncol. 2008;98:106-10.

11. Ha TK, An JY, Youn HG, Noh JH, Sohn TS, Kim S. Surgical outcome of synchronous second primary cancer in patients with gastric cancer. Yonsei Med J. 2007;48:981-7.

12. Hiyama T, Hanai A, Fujimoto I. Second primary cancer after diagnosis of stomach cancer in Osaka, Japan. Jpn J Cancer Res. 1991;82:762-70.

13. Hirono M, Niimoto M, Toge T, et al. A cohort study on second malignancies in gastrectomized patients with gastric cancer. II. Second malignancies in the gastric remnant. Jpn J Surg. 1986;16:344-50.

14. Lundegardh G, Hansson LE, Nyren O, Adami HO, Krusemo UB. The risk of gastrointestinal and other primary malignant diseases following gastric cancer. Acta Oncol. 1991;30:1-6.

15. Cheng TM. Taiwan's new national health insurance program: genesis and experience so far. Health Aff (Millwood). 2003;22:61-76.

16. Wu CW, Lo SS, Chen JH, Hsieh MC, Li AF, Lui WY. Multiple primary cancers in patients with gastric cancer. Hepatogastroenterology. 2006;53:463-7.

17. Park YK, Kim DY, Joo JK, et al. Clinicopathological features of gastric carcinoma patients with other primary carcinomas. Langenbecks Arch Surg. 2005;390:300-5.

18. Ikeda Y, Saku M, Kawanaka H, Nonaka M, Yoshida K. Features of second primary cancer in patients with gastric cancer. Oncology. 2003;65:113-7.

19. Dinis-Ribeiro M, Lomba-Viana H, Silva R, Moreira-Dias L, Lomba-Viana R. Associated primary tumors in patients with gastric cancer. J Clin Gastroenterol. 2002;34:533-5.

20. Buyukasik O, Hasdemir AO, Gulnerman Y, Col C, Ikiz O. Second primary cancers in patients with gastric cancer. Radiol Oncol. 2010;44:239-43.
21. Bozzetti F, Bonfanti G, Mariani L, Miceli R, Andreola S. Risk of second malignancies after surgical treatment for early gastric cancer. Gastric Cancer. 1999;2:74-5.

22. Ikeda Y, Saku M, Kishihara F, Maehara Y. Effective follow-up for recurrence or a second primary cancer in patients with early gastric cancer. Br J Surg. 2005;92:235-9.

23. Curtis RE FD, Ron E, Ries LAG, Hacker DG, Edwards BK, Tucker MA, Fraumeni JF Jr. (eds). New malignancies among cancer survivors: SEER cancer registries, 1973-2000. NIH publ. no. 05-5302. Bethesda: National Cancer Institute; 2006.

24. Kim SS, Ruiz VE, Carroll JD, Moss SF. Helicobacter pylori in the pathogenesis of gastric cancer and gastric lymphoma. Cancer Lett. 2011;305:228-38.

25. Williams CN. Helicobacter pylori, gastric cancer and gastric MALT lymphoma. Can J Gastroenterol. 1996;10:359-60.

26. Krishnan B, Morgan GJ. Non-Hodgkin lymphoma secondary to cancer chemotherapy. Cancer Epidemiol Biomark Prev. 2007; $16: 377-80$.

27. Mauch PM, Kalish LA, Marcus KC, et al. Second malignancies after treatment for laparotomy staged IA-IIIB Hodgkin's disease: long-term analysis of risk factors and outcome. Blood. 1996;87:3625-32.

28. Menu-Branthomme A, Rubino C, Shamsaldin A, et al. Radiation dose, chemotherapy and risk of soft tissue sarcoma after solid tumours during childhood. Int J Cancer. 2004;110:87-93.

29. Tucker MA, D'Angio GJ, Boice JD Jr, et al. Bone sarcomas linked to radiotherapy and chemotherapy in children. $\mathrm{N}$ Engl $\mathrm{J}$ Med. 1987;317:588-93.

30. Wong FL, Boice JD Jr, Abramson DH, et al. Cancer incidence after retinoblastoma. Radiation dose and sarcoma risk. JAMA. 1997;278:1262-7.

31. Henderson TO, Rajaraman P, Stovall M, et al. Risk factors associated with secondary sarcomas in childhood cancer survivors: a report from the childhood cancer survivor study. Int J Radiat Oncol Biol Phys. 2012;84:224-30.

32. Lo SF, Chang SN, Muo CH, et al. Modest increase in risk of specific types of cancer types in type 2 diabetes mellitus patients. Int J Cancer. 2013;132:182-8.

33. Kalaitzakis E, Gunnarsdottir SA, Josefsson A, Bjornsson E. Increased risk for malignant neoplasms among patients with cirrhosis. Clin Gastroenterol Hepatol. 2011;9:168-74.

34. Kornum JB, Svaerke C, Thomsen RW, Lange P, Sorensen HT. Chronic obstructive pulmonary disease and cancer risk: a Danish nationwide cohort study. Respir Med. 2012;106:845-52.

35. Takekuni K, Furukawa H, Tsukuma H, et al. The risk of second malignancy after adjuvant chemotherapy for stomach cancer. Gastric Cancer. 1999;2:206-9.

36. Kinoshita Y, Tsukuma H, Ajiki W, et al. The risk for second primaries in gastric cancer patients: adjuvant therapy and habitual smoking and drinking. J Epidemiol. 2000;10:300-4.

37. Nio Y, Hirahara N, Minari Y, et al. Second malignancies after a gastrectomy for gastric cancers: the effects of adjuvant therapies. Anticancer Res. 1999;19:3591-9. 